\title{
L-shaped fiber-chip grating couplers with high- directionality and low reflectivity fabricated with deep-UV lithography
}

\author{
Daniel Benedikovic, ${ }^{1}$ Carlos Alonso-Ramos, ${ }^{1, *}$ Diego PÉrez-GalaCho, ${ }^{1}$ \\ SylVAin Guerber, ${ }^{2}$ VladyslaV VAKARIN, ${ }^{1}$ GuIllaume MARCAUd, ${ }^{1}$ XAVIER Le \\ Roux, ${ }^{1}$ Eric Cassan, ${ }^{1}$ Delphine Marris-Morini, ${ }^{1}$ Pavel Cheben, ${ }^{3}$ Frédéric \\ Boeuf, ${ }^{2}$ Charles Baudot, ${ }^{2}$ And Laurent Vivien ${ }^{1}$ \\ ${ }^{1}$ Centre de Nanoscience et de Nanotechnology, CNRS, Univ. Paris-Sud, Université Paris-Saclay, C2N-Orsay, 91405 Orsay Cedex, France \\ ${ }^{2}$ TR\&D - STMicroelectronics SAS - 850 rue Jean Monnet - 38920 Crolles France \\ ${ }^{3}$ National Research Council Canada, 1200 Montreal Road, Ottawa, Ontario K1AOR6, Canada \\ *Corresponding author: carlos.ramos@u-psud.fr
}

Received XX Month XXXX; revised XX Month, XXXX; accepted XX Month XXXX; posted XX Month XXXX (Doc. ID XXXXX); published XX Month XXXX

\begin{abstract}
Grating couplers enable position-friendly interfacing of silicon chips by optical fibers. The conventional coupler designs call upon comparatively complex architectures to afford efficient light coupling to sub-micron siliconon-insulator (SOI) waveguides. Conversely, the blazing effect in double-etched gratings provides high coupling efficiency, with reduced fabrication intricacy. In this work, we demonstrate for the first time the realization of an ultra-directional L-shaped grating coupler, seamlessly fabricated by using 193-nm deep-ultraviolet (deep-UV) lithography. We also include a subwavelength index engineered waveguide-to-grating transition that provides an eight-fold reduction of the grating reflectivity, down to $1 \%(-20 \mathrm{~dB})$. A measured coupling efficiency of $-2.7 \mathrm{~dB}(54 \%)$ is achieved, with a bandwidth of $62 \mathrm{~nm}$. These results open promising prospects for implementation of efficient, robust, and cost-effective coupling interfaces for sub-micrometric SoI waveguides, as desired for large-volume applications in silicon photonics.
\end{abstract}

OCIS codes: (050.0050) Diffraction and gratings; (130.0130) Integrated optics; (050.1950) Diffraction gratings; (050.6624) Subwavelength structures.

http://dx.doi.org/10.1364/OL.99.099999

Silicon-on-insulator (SOI) has become a compelling material platform to realize nano-scale photonic devices, leveraging existing facilities of the microelectronic industry [1, 2]. However, the high confinement in SOI waveguides of sub- micrometric dimensions imposes a fundamental challenge for low-loss light coupling to or from access ports [3-25].

Optical input/output interfaces between optical fibers and SOI waveguides affording robustness, cost-effectivity, and high-efficiency coupling are widely recognized as a key functionality for large-volume applications. Surface grating couplers play essential role for such widespread deployment. They allow flexible positioning on the chip, thereby enabling automated wafer-scale testing, preferred for mass-scale scenarios. Furthermore, these devices generally provide larger tolerances in fabrication and alignment processes, compared to edge coupling counterparts [3-5].

Grating couplers, with an out-of-plane coupling configuration, are particularly unique components of the photonic device library, since they inherently desire high vertical index contrast to provide outstanding coupling reliance. However, the fiber-chip coupling efficiency is limited by the back-reflections at the grating-to-waveguide interface, mode mismatch between the Gaussian-like fiber mode and the exponential-like beam diffracted by the grating, and the power radiated towards the Si handle. The first two issues can be addressed by grating apodization [68]. However, the limited diffraction efficiency towards the fiber (directionality), which solely dictates the overall coupling efficiency, remains a significant challenge.

Typically, the improvement of the grating directionality may be achieved by exploiting the thin-film interference effect $[5,12]$. Further techniques for enhancing the directionality include additional overlayers [13], backside metallization [12, 17, 18], distributed Bragg mirrors 
underneath $[3,4,6,11]$, or multi-level grating architectures $[19,20]$ or non-standard etching depths [14-16, 23]. However, these approaches result in comparatively complex structures, which in turn, come with the expense of extra costs. Alternatively, it has been shown that the blazing effect may be exploited to achieve remarkably high directionalities [20-27]. Nevertheless, in many cases, high-efficiency grating couplers were fabricated using electron beam (e-beam) lithography. Albeit proven useful, particularly for concept demonstration, e-beam lithography is not compatible with large-volume industrially-driven applications. On the other hand, recent works suggest that moving towards thicker Si layers, e.g. $300 \mathrm{~nm}$, may be advantageous in terms of grating directionality $[3,5-7,10]$, yet enhancing the performance of other passive and active photonic devices [11].

In this work, we report, to the best of our knowledge, for the first time, on the experimental demonstration of an ultra-directional fiber-chip grating coupler seamlessly fabricated by using 193-nm deep-ultraviolet (deep-UV) optical lithography, viable tool for large-volume production. The grating coupler is implemented on a 300 -nm-thick Si layer in $300 \mathrm{~mm}$ SOI wafer, relying on standard full (300 $\mathrm{nm})$ and shallow (150 $\mathrm{nm})$ etch steps. The proposed Lshaped geometry (see Fig. 1) yields a remarkably high grating directionality exceeding 98\%, with a simplified fabrication process, compared with previously reported blazed gratings $[25,26]$. A coupling efficiency of $-2.7 \mathrm{~dB}$ is experimentally demonstrated, with a 3-dB bandwidth of 62 $\mathrm{nm}$, while a subwavelength index engineered transition provides an eight-fold reduction of the measured reflectivity, in particular reflectivity decrease from $\sim 8 \%$ to $\sim 1 \%$.

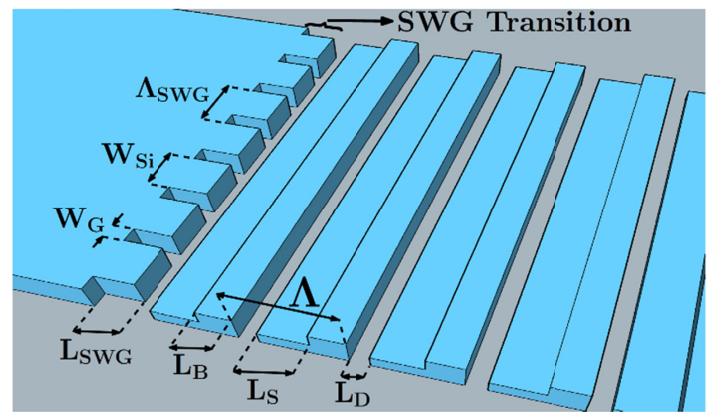

Fig. 1. Schematic view of proposed L-shaped fiber-chip grating coupler with sub-wavelength transition stage.

The schematic view of proposed L-shaped fiber-chip grating coupler with subwavelength transition is shown in Fig. 1. The grating period $(\Lambda)$ comprises deep- and shallow-etch trenches of lengths $L_{\mathrm{D}}$ and $L_{\mathrm{S}}$, respectively, and unetched Si block of length $L_{\mathrm{B}}$. Here, the deep- and shallow-etch levels are $300-\mathrm{nm}$ and $150-\mathrm{nm}$. The grating is optimized for transverse electrical (TE) polarization, operating in the Cband near $1.55 \mu \mathrm{m}$. Designs are carried out using 2-D Fourier-based expansion simulator [28] and threedimensional (3-D) finite difference time domain (FDTD) tool from Lumerical [29].

The L-shaped grating arrangement advantageously exploits the blazing effect for an outstanding ultra- directional performance, maximizing the diffraction towards the superstrate (upper cladding), while minimizing the power radiated into the Si handle.
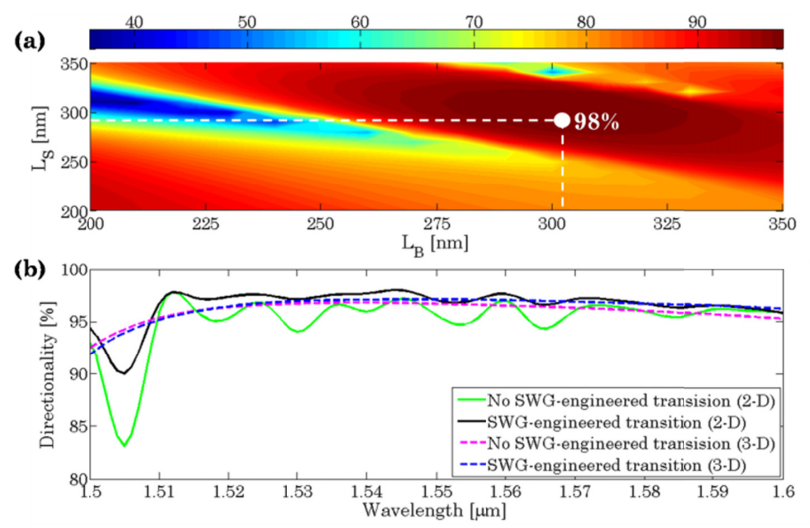

Fig. 2. (a) 2-D contour map of the directionality as a function of shallow- and non-etched grating segments, with $L_{\mathrm{D}}=120 \mathrm{~nm}$. (b) 2-D and 3-D calculations of the directionality as a function of a wavelength for nominal grating coupler designs with and without subwavelength transition.

First, we optimized dimensions of the grating coupler without the subwavelength transition stage. Figure 2(a) shows the 2-D calculations of the directionality as a function of the length of the shallow-etched $\left(L_{S}\right)$ and non-etched $\left(L_{B}\right)$ grating segments, for a fixed length of deep etched grating part of $L_{\mathrm{D}}=120 \mathrm{~nm}$. From this analysis, we chose grating dimensions of $L_{\mathrm{D}}=120 \mathrm{~nm}, L_{\mathrm{S}}=290 \mathrm{~nm}$ and $L_{\mathrm{B}}=310 \mathrm{~nm}$, providing a directionality of $98 \%$. It became apparent that a wide range of geometries provided high directionality, suggesting a large tolerance to fabrication errors. As shown in Fig. 2(b), the proposed design yields a directionality higher than $90 \%$ in a wide spectral range of $100 \mathrm{~nm}$.

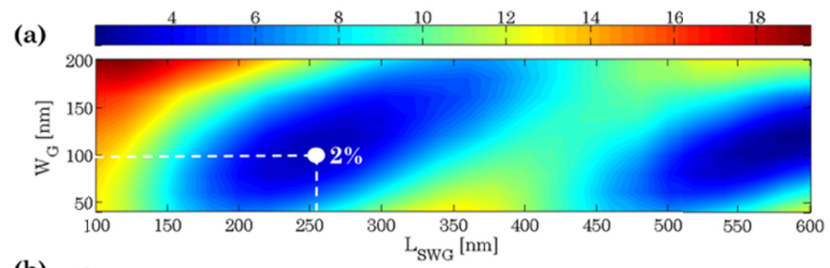

(b)

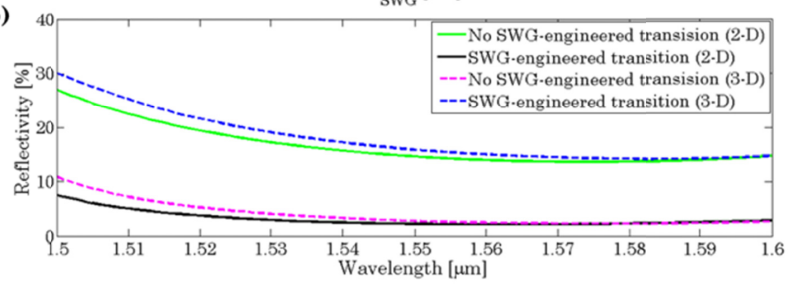

Fig. 3. (a) 2-D contour map of the reflectivity as a function of the length of the transition stage length and the width of SWG holes. (b) 2-D and 3-D calculations of the reflectivity as a function of wavelength for nominal grating coupler designs with and without subwavelength transition.

We implemented a metamaterial transition stage between the injection waveguide and the grating to reduce back- 
reflections. This has been realized by exploiting the concept of sub-wavelength grating (SWG) structuration, as demonstrated for the first time by Cheben et al. [30-32], and more recently extensively used in a variety of photonic devices [33].

As depicted in Fig. 1, the SWG antireflection stage is implemented along the transversal direction, i.e. perpendicular to the light propagation. We design section length $\left(L_{S W G}\right)$ and the width of the gaps $\left(W_{G}\right)$ and Si pillars $\left(W_{S i}\right)$ to minimize back-reflections. Figure 3(a) shows the reflectivity, calculated with 3-D FDTD, as a function of the gap width and section length, for a fixed transversal pitch of $\Lambda_{\text {sWG }}=400 \mathrm{~nm}$. Back-reflections can be minimized, for different SWG configurations, proving the flexibility of the proposed approach. In Fig. 3(b), we compare the reflectivity spectrum of the grating with and without antireflection stage, calculated with 3-D FDTD and 2-D Fourier-based methods. Here, we considered $L_{S W G}=255 \mathrm{~nm}$ and $W_{G}=100$ $\mathrm{nm}$. The proposed subwavelength transition stage yields an eight-fold reduction in reflectivity, from $16 \%$ to $2 \%$ at 1550 $\mathrm{nm}$ wavelength. Furthermore, this transition stage minimizes back-reflections in the waveguide-grating interface in a wideband range exceeding $100 \mathrm{~nm}$. Note that the increase in reflectivity for shorter wavelengths, occurring for grating with and without subwavelength stage, is mainly produced by the proximity of the $2^{\text {nd }}$ order Bragg condition.
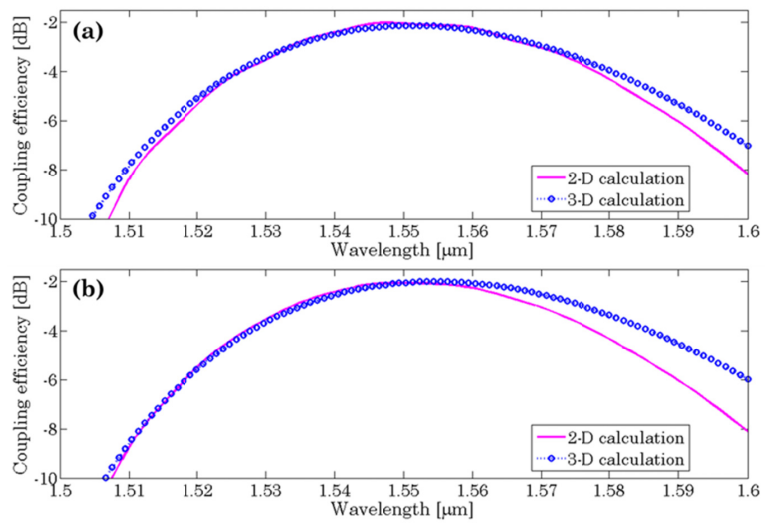

Fig. 4. 2-D and 3-D calculations of the coupling efficiency as a function of a wavelength for nominal grating coupler designs: (a) without and (b) with SWG-engineered transition.

Interestingly, this back-reflection cancelation approach does not affect the grating directionality. According to our simulations, the grating with subwavelength transition has a directionality higher than $98 \%$ [see Fig. 2(b)]. We calculated the coupling efficiency for both grating coupler designs, with and without subwavelength stage. As shown in Fig. 4, calculations predict a coupling efficiency of around $-2.2 \mathrm{~dB}$ for both designs, with a coupling angle of $22^{\circ}$. In the case of the grating without subwavelength transition, the overlap between radiated field and fiber mode is close to $80 \%$. For the structure with SWG transition the overlap value falls to a $60 \%$. This effect counteracts the lower back-reflections in the structure with subwavelength transition and accounts for the similar coupling efficiency. Apodization techniques could improve the overlap value, allowing to fully exploit the potential of the proposed approach. Still, the simplicity of the subwavelength transition makes it very interesting for applications requiring low back-reflections.

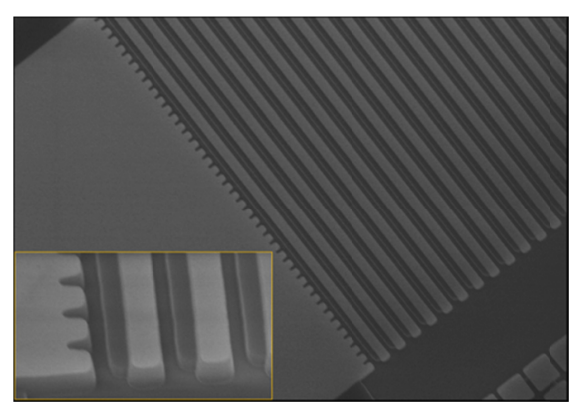

Fig. 5. Scanning electron microscopy image of the fabricated grating couplers. Inset: Close-up view on the grating geometry.

Surface grating couplers were fabricated using $300 \mathrm{~mm}$ SOI photonic platform with $193 \mathrm{~nm}$ deep-UV optical lithography. The vertical dimensions comprise 300-nm-thick $\mathrm{Si}$ film on top of $720 \mathrm{~nm}$ oxide layer. The double-etch process was used to define shallow and deep etched grating trenches as well as the interconnecting strip waveguides. The grating couplers were $15 \mu \mathrm{m}$ wide and $21 \mu \mathrm{m}$ long, and were connected to single-mode waveguides by $500-\mu \mathrm{m}$-long tapers. Finally, they were covered by a silicon dioxide layer for protection. The scanning electron microscopy (SEM) images of a fabricated surface grating coupler, before silica cladding deposition are shown in Fig. 5.

Cleaved single-mode optical fibers (SMF-28) have been used as access ports at the input and the output of the Si chip. The coupling efficiency have been determined from the insertion loss measurements of two identical grating couplers arranged in a back-to-back configuration.

The measured fiber-chip coupling efficiencies of fabricated devices, with and without subwavelength transition stage, are shown in Fig. 6(a). Specifically, an experimental peak coupling efficiency of $-3.4 \mathrm{~dB}$ near the wavelength of 1560 $\mathrm{nm}$, with a 3-dB bandwidth of $46 \mathrm{~nm}$, is achieved for a grating coupler design without the SWG transition. Here, the spectral response of the grating coupler exhibits noticeable Fabry-Perot ripples. From the amplitude of these ripples, that varies between $0.7 \mathrm{~dB}$ and $0.85 \mathrm{~dB}$, with a periodicity of $\sim 0.26 \mathrm{~nm}$ [see Fig. 6(b)], we estimated a reflectivity of $\sim 8 \%$. The origin of these ripples is attributed to the backreflections due to the large discontinuity at the waveguideto-grating transition. Conversely, for the grating coupler with a SWG transition, the measured coupling efficiency reaches the peak of $-2.7 \mathrm{~dB}$ at a wavelength of $1565 \mathrm{~nm}$, with a bandwidth of $62 \mathrm{~nm}$. The grating coupler response still shows some residual fringes, yet of comparatively smaller magnitude up to $0.1 \mathrm{~dB}$. This yields to an estimated grating reflectivity of $\sim 1 \%$. This is an eight-fold reduction in measured back-reflection. 

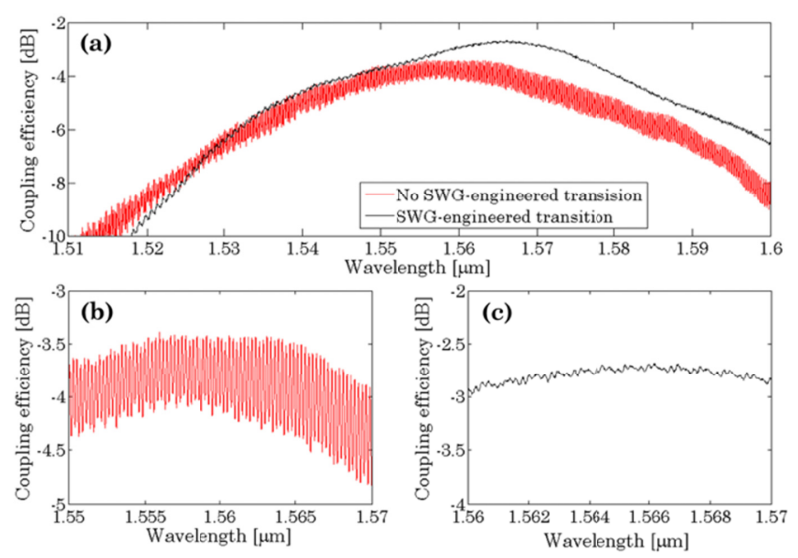

Fig. 6. (a) Measured fiber-chip coupling efficiency as a function of wavelength for dual-etch grating couplers. Detail view on spectral response of grating couplers: (b) without and (c) with SWG-engineered metamaterial transition.

In summary, we report, to the best of our knowledge, for the first time, on the experimental demonstration of highdirectionality fiber-chip grating coupler with low reflectivity, realized with $193 \mathrm{~nm}$ deep-UV optical lithography. The fabricated devices yield a measured fiber-chip coupling efficiency of $-2.7 \mathrm{~dB}$ and $3-\mathrm{dB}$ bandwidth of $62 \mathrm{~nm}$. Furthermore, we experimentally demonstrated that a SWG transition stage effectively suppressed the reflectivity down to $1 \%$, yielding an eight-fold improvement compared to the regular double-etch blazed grating coupler. These results prove the compatibility of the high-directionality L-shaped gratings with large-volume fabrication processes, paving the way towards the deployment of high-performance and costeffective fiber-chip coupling interfaces in a large-volume photonic applications.

Funding. H2020 European Research Council (ERC) (ERC POPSTAR 647342); the NANO2017 programme with STMicroelectronics funded by the "Ministère de l'économie, de l'industrie et du numérique", "Délégation Générales des Entreprises".

\section{References}

1. T. Baehr-Jones, T. Pinguet, P. L. Guo-Qiang, S. Danziger, D. Prather, and M. Hochberg, Nat. Photonics 6, 206 (2012).

2. M. Hochberg and T. Baehr-Jones, Nat. Photonics 4, 492 (2010).

3. A. Mekis, S. Gloeckner, G. Masini, A. Narasimha, T. Pinguet, S. Sahni, and P. De Dobbelaere, IEEE J. Sel. Quantum Electron. 17, 597 (2011).

4. C. Kopp, S. Bernabe, B. Bakir, J.M. Fédéli, R. Orobtchouk, F. Schrank, H. Porte, L. Zimmermann, and T. Tekin, IEEE J. Sel. Top. Quantum Electron. 17, 498 (2011).

5. D.-X. Xu, J. H. Schmid, G. T. Reed, G. Z. Mashanovich, D. J. Thomson, M. Nedeljkovic, X. Chen, D. Van Thourhout, S. Keyvaninia, and S. K. Selvaraja, IEEE J. Sel. Top. Quantum Electron. 20, 189 (2014).

6. Ch. Baudot, D. Dutartre, A. Souhaité, N. Vulliet, A. Jones, M. Ries, A. Mekis, L. Verslegers, P. Sun, Y. Chi, S. Cremer, O. Gourhant, D. Benoit, G. Courgoulet, C. Perrot, L. Broussous, T. Pinguet, J. Sinviant, and F. Boeuf, in Proceeding of IEEE $11^{\text {th }}$ International Conference on Group IV Photonics (IEEE, 2014), pp. 137-138.
7. X. Chen, Ch. Li, Ch. K. Y. Fung, S. M. G. Lo, and H. K. Tsang, IEEE Photon. Technol. Lett. 22, 1156 (2010).

8. R. Halir, P. Cheben, J. H. Schmid, R. Ma, D. Bedard, S. Janz, D.-X. Xu, A. Densmore, J. Lapointe, and I. Molina-Fernández, Opt. Lett. 35, 3243-3245 (2010).

9. R. Halir, R. Halir, P. Cheben, S. Janz, D.-X. Xu, Í. Molina-Fernández, and J. G. Wangüemert-Pérez, Opt. Lett. 34, 1408 (2009).

10.A. Bozzola, L. Carroll, D. Gerace, I. Cristiani, and L. C. Andreani, Opt. Express 23, 16289 (2015).

11.D. Marris-Morini, L. Virot, C. Baudot, J.-M. Fédéli, G. Rasigade, D. Perez-Galacho, J.-H. Hartmann, S. Oliver, P. Brindel, P. Crozat, F. Boeuf, and L. Vivien, Opt. Express 22, 6674 (2014).

12.D. Benedikovic, P. Cheben, J. H. Schmid, D.-X. Xu, B. Lamontagne, S. Wang, J. Lapointe, R. Halir, A. Ortega-Moñux, S. Janz, and M. Dado, Opt. Express 23, 22628 (2015).

13.D. Vermeulen, S. Selvaraja, P. Verheyen, G. Lepage, W. Bogaerts, P. Absil, D. Van Thourhout, and G. Roelkens, Opt. Express 18, 18278 (2010).

14.C. Li, H. Zhang, M. Yu, and G. Q. Lo, Opt. Express 21, 7868 (2013).

15.X. Wen, K. Xu, and Q. Song, Photonics Research 4, 209 (2016).

16.C. A. Flory, IEEE J. Quantum Electron. 40, 949 (2004).

17.W. S. Zaoui, A. Kunze, W. Vogel, M. Berroth, J. Butschke, F. Letzkus, and J. Burhhartz, Opt. Express 22, 1277 (2014).

18.Y. Ding, C. Peucheret, H. Ou, and K. Yvind, Op. Lett. 39, 5348 (2014).

19.W. D. Sacher, Y. Huang, L. Ding, B. J. F. Taylor, H. Jayatilleka, G.-Q. Lo, and J. K. S. Poon, Opt. Express 22, 10938 (2014).

20.J. Notaros, F. Pavanello, M. T. Wade, C. Gentry, A. Atabaki, L. Alloatti, R. J. Ram, and M. Popovic, in Optical Fiber Communication Conference, OSA Technical Digest (Optical Society of America, 2016), paper M2I.5.

21.T. Tamir and S, T. Peng, Appl. Phys. 14, 235 (1977).

22.T. Aoyagi, Y. Aoyagi, and S. Namba, Appl. Phys. Lett. 29, 303 (1976).

23.M. Fan, M. A. Popovic, and F. X. Kartner, in Conference on Lasers and Electro-Optics/Quantum Electronics and Laser Science Conference and Photonics Applications Systems Technologies (Optical Society of America, 2007), paper CTuDD3.

24.Y. Chen, R. Halir, I. Molina-Fernandez, P. Cheben, and J.-J. He, Opt. Lett. 41, 5059 (2016).

25.C. Alonso-Ramos, P. Cheben, A. Ortega-Moñux, J. H. Schmid, D.-X. Xu, and I. Molina-Fernández, Opt. Lett. 39, 5351 (2014).

26.D. Benedikovic, C. Alonso-Ramos, P. Cheben, J. H. Schmid, S. Wang, D.-X. Xu, J. Lapointe, S. Janz, R. Halir, A. Ortega-Moñux, J. G. Wangüemert-Perez, I. Molina-Fernandez, J.-M. Fédéli, L. Vivien, and M. Dado, Opt. Lett. 40, 4190 (2015).

27.T. Watanabe, M. Ayata, U. Koch, Y. Fedoryshyn, and J. Leuthold, in Conference on Lasers and Electro-Optics, OSA technical Digest (Optical Society of America, 2017), paper SF1I.2.

28.L. Zavargo-Peche, A. Ortega-Moñux, J. G. Wangüemert-Pérez, and I. Molina-Fernández, Prog. Electromagn. Res. 123, 447 (2012).

29.Lumerical Solutions, Inc., http://www.lumerical.com.

30.P. Cheben, D.-X. Xu, S. Janz, and A. Densmore, Opt. Express 14, 4695-4702 (2006).

31.P. J. Bock, P. Cheben, J. H. Schmid, J. Lapointe, A. Delage, S. Janz, G. C. Aers, D.-X. Xu, A. Densmore, and T. J. Hall, Opt. Express 18, 20251-20262 (2010).

32.P. Cheben, P. J. Bock, J. H. Schmid, J. Lapointe, S. Janz, D.-X. Xu, A. Densmore, A. Delage, B. Lamontagne, and T. J. Hall, 35, 2526-2528 (2010).

33.R. Halir, P. Bock, P. Cheben, A. Ortega-Moñux, C. Alonso-Ramos, J. H. Schmid, J. Lapointe, D.-X. Xu, J. G. Wangüemert-Pérez, I. MolinaFernández, and S. Janz, Laser Photon. Rev. 9, 25-49 (2015). 


\section{Full references}

1. T. Baehr-Jones, T. Pinguet, P. L. Guo-Qiang, S. Danziger, D. Prather, and M. Hochberg, "Myths and rumors of silicon photonics," Nat. Photonics 6(4), 206 (2012).

2. M. Hochberg and T. Baehr-Jones, "Towards fabless silicon photonics," Nat. Photonics 4(8), 492-494 (2010).

3. A. Mekis, S. Gloeckner, G. Masini, A. Narasimha, T. Pinguet, S. Sahni, and P. De Dobbelaere, "A grating-coupler-enabled CMOS photonics platform," IEEE J. Sel. Quantum Electron. 17(3), 597-608 (2011).

4. C. Kopp, S. Bernabe, B. Bakir, J.M. Fedeli, R. Orobtchouk, F. Schrank, H. Porte, L. Zimmermann, and T. Tekin, "Silicon photonic circuits: On-CMOS integration, fiber optical coupling, and packaging," IEEE J. Sel. Top. Quantum Electron. 17(3), 498-509 (2011).

5. D.-X. Xu, J. H. Schmid, G. T. Reed, G. Z. Mashanovich, D. J. Thomson, M. Nedeljkovic, X. Chen, D. Van Thourhout, S. Keyvaninia, and S. K. Selvaraja, "Silicon photonic integration platform - Have we found the sweet spot?," IEEE J. Sel. Top. Quantum Electron. 20(4), 189-205 (2014).

6. Ch. Baudot, D. Dutartre, A. Souhaité, N. Vulliet, A. Jones, M. Ries, A. Mekis, L. Verslegers, P. Sun, Y. Chi, S. Cremer, O. Gourhant, D. Benoit, G. Courgoulet, C. Perrot, L. Broussous, T. Pinguet, J. Sinviant, F. Boeuf, "Low cost $300 \mathrm{~mm}$ double-SOI substrate for low insertion loss 1D \& 2D grating couplers," in Proceeding of IEEE $11^{\text {th }}$ International Conference on Group IV Photonics (IEEE, 2014), pp. 137-138.

7. X. Chen, Ch. Li, Ch. K. Y. Fung, S. M. G. Lo, H. K. Tsang, "Apodized waveguide grating couplers for efficient coupling to optical fibers," IEEE Photonic Tech L 22(15), 1156-1158 (2010).

8. R. Halir, P. Cheben, J. H. Schmid, R. Ma, D. Bedard, S. Janz, D.-X. Xu, A. Densmore, J. Lapointe, and I. Molina-Fernández, "Continuously apodized fiber-to-chip surface grating coupler with refractive index engineered subwavelength structure," Opt. Lett. 35(19), 3243-3245 (2010).

9. R. Halir, R. Halir, P. Cheben, S. Janz, D.-X. Xu, Í. Molina-Fernández, and J. G. Wangüemert-Pérez, "Waveguide grating coupler with subwavelength microstructures," Opt. Lett. 34(9), 1408-1410 (2009).

10.A. Bozzola, L. Carroll, D. Gerace, I. Cristiani, and L. C. Andreani, "Optimising apodized grating couplers in a pure SOI platform to -0.5 dB coupling efficiency," Opt. Express 23(12), 16289-16304 (2015).

11.D. Marris-Morini, L. Virot, C. Baudot, J.-M. Fédéli, G. Rasigade, D. Perez-Galacho, J.-H. Hartmann, S. Oliver, P. Brindel, P. Crozat, F. Boeuf, and L. Vivien, "A 40 Gbit/s optical link on a 300-mm silicon platform", Opt. Express 22(6), 6674-6679 (2014).

12.D. Benedikovic, P. Cheben, J. H. Schmid, D.-X. Xu, B. Lamontagne, S. Wang, J. Lapointe, R. Halir, A. Ortega-Moñux, S. Janz, and M. Dado, "Subwavelength index engineered surface grating coupler with subdecibel efficiency for 220-nm silicon-on-insulator waveguides", Opt. Express 23(17), 22628-22635 (2015).

13.D. Vermeulen, S. Selvaraja, P. Verheyen, G. Lepage, W. Bogaerts, P. Absil, D. Van Thourhout, and G. Roelkens, "High efficiency fiber-tochip grating couplers realized using an advanced CMOS-compatible silicon-on-insulator platform," Opt. Express 18(17), 18278-18283 (2010).

14.C. Li, H. Zhang, M. Yu, and G. Q. Lo, "CMOS-compatible high efficiency double-etched apodized waveguide grating coupler," Opt. Express 21(7), 7868-7874 (2013).

15.X. Wen, K. Xu, and Q. Song, "Design of a barcode-like waveguide nanostructure for efficient chip-fiber coupling,"Photonics Research 4(6), 209-2013 (2016).

16.C. A. Flory, "Analysis of directional grating-coupled radiation in waveguide structures," IEEE J. Quantum Electron. 40(7), 949-957 (2004).
17.W. S. Zaoui, A. Kunze, W. Vogel, M. Berroth, J. Butschke, F. Letzkus, and J. Burghartz, "Bridging the gap between optical fibers and silicon photonic integrated circuits," Opt. Express 22(2), 1277-1286 (2014).

18.Y. Ding, C. Peucheret, H. Ou, and K. Yvind, "Fully etched apodized grating coupler on the SOI platform with $-0.58 \mathrm{~dB}$ coupling efficiency," Op. Lett. 39(18), 5348-5350 (2014).

19.W. D. Sacher, Y. Huang, L. Ding, B. J. F. Taylor, H. Jayatilleka, G.-Q. Lo, and J. K. S. Poon, "Wide bandwidth and high coupling efficiency $\mathrm{Si}_{3} \mathrm{~N}_{4}$-on-SOI dual-level grating coupler," Opt. Express 22(9), 1093810947 (2014).

20.J. Notaros, F. Pavanello, M. T. Wade, C. Gentry, A. Atabaki, L. Alloatti, R. J. Ram, and M. Popovic, "Ultra-efficient CMOS fiber-to-chip grating couplers," in Optical Fiber Communication Conference, OSA Technical Digest (Optical Society of America, 2016), paper M2I.5.

21.T. Tamir and S, T. Peng, "Analysis and design of grating couplers," Appl. Phys. 14(3), 235-254 (1977).

22.T. Aoyagi, Y. Aoyagi, and S. Namba, "Highefficiency blazed grating couplers," Appl. Phys. Lett. 29(5), 303-304 (1976).

23.M. Fan, M. A. Popovic, and F. X. Kartner, "High directivity, fiber-tochip coupler with anisotropically radiating grating teeth," in Conference on Lasers and Electro-Optics/Quantum Electronics and Laser Science Conference and Photonic Applications Systems Technologies (Optical Society of America, 2007), paper CTuDD3.

24.Y. Chen, R. Halir, I. Molina-Fernandez, P. Cheben, and J.-J. He, "High efficiency apodized-imaging fiber-chip grating coupler for silicon nitride waveguides," Opt. Lett. 41(21), 5059-5062 (2016).

25.C. Alonso-Ramos, P. Cheben, A. Ortega-Moñux, J. H. Schmid, D.-X. $\mathrm{Xu}$, and I. Molina-Fernández, "Fiber-chip grating coupler based on interleaved tranches with directionality exceeding 95\%," Opt. Lett. 39(18), 5351-5354 (2014).

26.D. Benedikovic, C. Alonso-Ramos, P. Cheben, J. H. Schmid, S. Wang, D.-X. Xu, J. Lapointe, S. Janz, R. Halir, A. Ortega-Moñux, J. G. Wangüemert-Pérez, I. Molina-Fernandez, J.-M. Fédéli, L. Vivien, and M. Dado, "High-directionality fiber-chip grating coupler with interleaved trenches and subwavelength index-matching structure," Opt. Lett. 40(18), 4190-4193 (2015).

27.L. Zavargo-Peche, A. Ortega-Moñux, J. G. Wangüemert-Pérez, and I. Molina-Fernández, "Fourier based combined techniques to design novel sub-wavelength optical integrated devices," Prog. Electromagn. Res. 123, 447-465 (2012).

28.Lumerical Solutions, Inc., http://www.lumerical.com.

29.P. Cheben, D.-X. Xu, S. Janz, and A. Densmore, "Subwavelength waveguide grating for mode conversion and light coupling in integrated optics," Opt. Express 14(11), 4695-4702 (2006).

30.P. J. Bock, P. Cheben, J. H. Schmid, J. Lapointe, A. Delage, S. Janz, G. C. Aers, D.-X. Xu, A. Densmore, and T. J. Hall, "Subwavelength grating periodic structures in silicon-on-insulator: a new type of microphotonic waveguide," Opt. Express 18(19), 20251-20262 (2010).

31.P. Cheben, P. J. Bock, J. H. Schmid, J. Lapointe, S. Janz, D.-X. Xu, A. Densmore, A. Delage, B. Lamontagne, and T. J. Hall, "Refractive index engineering with subwavelength gratings for efficient microphotonic couplers and planar waveguide multiplexers," 35(15), 2526-2528 (2010).

32.R. Halir, P. Bock, P. Cheben, A. Ortega-Monux, C. Alonso-Ramos, J. H. Schmid, J. Lapointe, D.-X. Xu, J. G. Wangüemert-Pérez, I. MolinaFernández, and S. Janz, "Waveguide sub-wavelength structures: a review of principles and applications," Laser Photon. Rev. 9(1), 25-49 (2015). 